\title{
Relación entre Calidad de Vida en el Trabajo y Síntomas de Estrés en el Personal Administrativo Universitario
}

\section{RELATIONSHIP BETWEEN QUALITY OF LIFE AT WORK AND STRESS SYMPTOMS IN UNIVERSITY ADMINISTRATIVE STAFF}

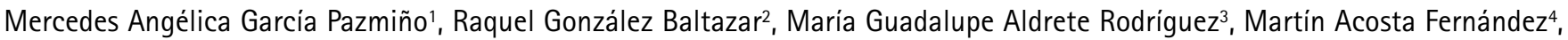 \\ Silvia Graciela León Cortés ${ }^{5}$ \\ 1. Doctora en Psicología Clínica, Máster en Psicología Deportiva, docente de la Universidad Central del Ecuador. Quito-Ecuador. \\ 2. Dra. Miembro del Instituto de Investigación en Salud Ocupacional, Departamento de Salud Pública, Centro Universitario de Ciencias de la Salud, U de \\ Guadalajara.
}

3. Dra. Investigador: Instituto de Investigación en Salud Ocupacional (IISO) Universidad de Guadalajara.

4. Dr. Investigador: Instituto de Investigación en Salud Ocupacional (IISO) Universidad de Guadalajara.

5. Dra. Investigador: Instituto de Investigación en Salud Ocupacional (IISO) Universidad de Guadalajara.

\begin{abstract}
RESUMEN
Objetivo: Analizar la relación de la Calidad de Vida en el Trabajo (CVT), con los síntomas del estrés en el personal administrativo de una universidad pública del Ecuador. Material y métodos: Diseño correspondiente con el paradigma cuantitativo, transversal, analítico y relacional. Resultados: Del análisis descriptivo de la muestra de 447 trabajadores, 59.3\% mujeres y 40.7\% hombres; promedio de edad es 46 años. Tiempo promedio en el cargo es 18 años. Se aplicó cuestionarios: "CVT-GOHISALO" y "Evaluación de Síntomas de Estrés". El $76 \%$ de los trabajadores administrativos advierte la presencia de los síntomas fisiológicos de estrés en niveles medio, alto y muy alto. A su vez los síntomas de comportamiento social, intelectuales, laborales y psicoemocionales presentaron niveles bajos de estrés. No hay presencia de trastornos mentales o psicoemocionales. Más de la mitad de la población estudiada estaría en vulnerabilidad al riesgo del desequilibrio en la CVT; la tercera parte de la población estudiada obtuvo una alta satisfacción en el trabajo. Conclusión: Este estudio fue inédito, demostró la asociación significativa entre la CVT y los síntomas de estrés. Los resultados permitieron plantear estrategias predictivas para mejorar la CVT en el contexto universitario; alineándose a las políticas de Estado, promover el "buen vivir".
\end{abstract}

(García M, González R, Aldrete M, Acosta M, León S, 2014. Relación entre Calidad de Vida en el Trabajo y Síntomas de Estrés en el Personal Administrativo Universitario. Cienc Trab. May-Ago; 16 [50]: 97-102).

Palabras claves : CALIDAD DE VIDA EN EL TRABAJO, SÍNTOMAS DE ESTRÉS, SATISFACCIÓN EN EL TRABAJO.

\section{ABSTRACT}

Objective: To analyze the relationship of the Quality of Life at Work (CVT) with symptoms of stress on the administrative staff of a public university in Ecuador. Materials and methods: Design corresponding to the quantitative, cross-sectional, analytical and relational paradigm. Results: Descriptive analysis of the sample of 447 workers in total, $59.3 \%$ female and $40.7 \%$ male; average age is 46 years old. The average time in office is 18 years. Questionnaires were applied: "CVT-GOHISALO" and "Assessment of Stress Symptoms" 76\% of administrative workers, admit to the presence of the physiological symptoms of stress in the medium, high and very high levels. In turn the symptoms of social, intellectual, occupational and psycho-emotional behavior, had low levels of stress. No presence of mental or psycho-emotional disorders. More than half of the studied population would be vulnerable to the risk of imbalance in the quality of life at work. third of the study population, had a high job satisfaction. Conclusion: This study was unpublished and showed a significant association between CVT and symptoms of stress. The results allowed to propose strategies to improve predictive quality of life at work in the university context; aligning state policies to promote the "good life".

Key words: QUALITY OF LIFE AT WORK, SYMPTOMS OF STRESS, JOB SATISFACTION.

\section{INTRODUCCIÓN}

¿Cómo se relaciona la calidad de vida en el trabajo con los síntomas de estrés en el personal administrativo de una univer-

\section{Correspondencia / Correspondence:}

Mercedes Angélica Garcia Pazmiño

Calle Francisco Salamba OE2-220 y Juan Clamavea. Quito-Ecuador

Tel.: 593-99 4828597, 59322479404

e-mail: garcia.mercy@hotmail.com

Recibido: 21 de Junio 2014 / Aceptado: 23 de Julio 2014 sidad pública?, fue la pregunta planteada que condujo la investigación. En toda época las personas han procurado buscar modelos de vida más saludables, ya sea en el ámbito educativo, económico, político, deportivo o filosófico, entre otros; y el medio para conseguirla ha sido a través del esfuerzo, las creencias, recreación, actividad física y especialmente el trabajo, pero el fin es llegar a un estado de bienestar., ${ }^{1,2}$

Por lo tanto, la calidad de vida en el trabajo (CVT) implica tener una participación proactiva en la solución de problemas y toma de decisiones organizacionales; se relaciona especialmente con el bienestar, satisfacción, seguridad, identificación e integración al puesto de trabajo, así como en la administración adecuada de su tiempo libre para armonizar vida laboral y familiar. ${ }^{3}$ 
El tema de la calidad de vida ha sido un tema atrayente a través del tiempo. La historia revela el afán de humanizar el entorno de trabajo, prestando mayor atención al factor humano y a la mejora de la calidad de vida; así se creó en los años setenta en Estados Unidos el "Movimiento de Calidad de Vida Laboral, CVL", según refiere ${ }^{4}$, el mismo que hacía alusión al "nivel de vida" y "bienestar psicológico". ${ }^{4}$ Entendiéndose el bienestar psicológico como el estado subjetivo que refleja el desarrollo de las capacidades y el crecimiento personal. $^{5}$

Asimismo, se aprecia una creciente concientización a nivel mundial sobre el estrés y la manifestación de sus síntomas, responsables directos de las enfermedades físicas, psíquicas y sociales más comunes que afectan al hombre. ${ }^{6}$

Los síntomas del estrés como la alteración de los ritmos biológicos, fisiológicos y psicológicos, inestabilidad, depresión, inseguridad e incertidumbre interfieren en la salud del trabajador y, a su vez, en el ambiente laboral; el aumento de los niveles de estrés dificulta la integración al trabajo y muchos profesionales experimentan frustración y un estado de tensión emocional. "Todos los trabajos son potencialmente estresantes, aunque las tensiones varian considerablemente, en función de cada individuo y forma de afrontamiento"?

Matteson e Ivancevich ${ }^{8}$ plantean que los síntomas de estrés causan el abandono del empleo como respuesta de huida, lo que determina un alto índice de rotación de personal (40\%). Este tema se ha difundido en los últimos tiempos de tal manera que se ha vuelto trillado, por tal motivo sus efectos pueden ser invisibilizados; sin embargo, está latente y es gradual. De la misma manera las características de la respuesta guardan una relación con aspectos individuales, sociales y culturales que dan un relieve particular a la experiencia percibida de cada persona. ${ }^{9}$

En forma paralela en Estados Unidos se busca mejorar la productividad, pero a partir del bienestar del trabajador, quien es partícipe activo en la toma de decisiones, en procura del desarrollo de competencias en el trabajador; el bienestar y la salud son indispensables para que se desempeñen óptimamente en sus tareas. Chiavenato ${ }^{10}$ complementa sugiriendo que el lugar de trabajo debe ser adecuado, acondicionado de tal forma que no solo insinúe respeto, sino también motivación y satisfacción al trabajador.

Para explicar la CVT, Nadler y Lawler ${ }^{11}$ plantean un modelo que describe la CVT, basados en cuatro características fundamentales: a) involucra la participación activa de los empleados en los asuntos laborales; b) la innovación en el sistema de recompensas de trabajo; c) el mejoramiento de las condiciones de trabajo, y d) la reestructuración de tareas delimitando las responsabilidades y roles de cada empleado. En el mismo sentido, la CVT se ha enfatizado como una filosofía para organizar y dirigir, donde los empleados son parte activa de la organización y es importante que experimenten la percepción de bienestar y satisfacción en sus trabajos. La generación del milenio se enfrenta al desafío del estrés y a la aplicación de nuevos recursos de afrontamiento, porque estamos en un cambio de era, que implica un cambio de paradigmas hacia una nueva cultura organizacional.

Es ineludible resaltar la crisis socio-económica y laboral en los años 90 al 2000, por la que atravesó el Ecuador; y en esa relación Estado-Universidad, el panorama nacional a nivel universitario también se afectó; pero la universidad pública, con una actitud resiliente, reflexionó y se posicionó de nuevas alternativas que favorecen a la calidad del sistema universitario, para configurar una respuesta propia, basada en el desarrollo y difusión de saberes científicos y universales para aplicarlos a la solución de los problemas de subdesarrollo económico y político que enfrenta el país, para lo cual refuerza las competencias de gestión de apoyo y recurso; es decir, perfecciona al talento humano, cambia sus métodos y técnicas para orientarlos hacia la investigación científica, tecnología y generación de empleo; para atender mejor al estudiantado, propone una reforma pedagógica y una sucesión docente.

El personal administrativo de una institución de educación superior debe ir a la par del desarrollo dinámico y productivo del mundo de hoy; por tal motivo es considerado como el talento humano que contribuye al desenvolvimiento de los procesos administrativos porque es la imagen institucional, justamente por estar en contacto, tanto con los usuarios internos y externos, como por ende las exigencias y la constante presión son parte de la cotidianidad del trabajo administrativo; a veces esta presión se hace excesiva o difícil de controlar. ${ }^{12}$

Actualmente las universidades públicas en el Ecuador se encuentran en un proceso de evaluación y acreditación que valora $\mathrm{y}$ promueve la calidad y rendición de cuentas a la sociedad por parte del Consejo de Evaluación, Acreditación y Aseguramiento de la Calidad de la Educación Superior (CEAACES). El presente estudio tiene relevancia y trascendencia en la medida en que se propone analizar la relación entre calidad de vida en el trabajo y los síntomas de estrés en el personal administrativo de una Universidad Pública. Considerando que la mayor parte de la población del mundo (58\%) pasa una tercera parte de su vida adulta laborando, busca aportar en mejorar la calidad de vida en el trabajo (CVT); justamente el carácter trascendental del proyecto se enmarcó en concienciar y contribuir a la calidad académica y administrativa de la universidad pública.

El fundamento teórico del estudio es el positivismo, cuyos postulados establecen que la razón debe ser aplicada por lo que perciben los sentidos, la experiencia sensorial y la conciencia. Y a su vez propende hacia un estado positivo del hombre, hacia la calidad de vida. Y confluye con la transformación social, en la cual se ha trascendido desde modelos económicos, de industrialización y desarrollo, donde se pretendió la humanización del trabajo, donde el empleado es el articulador del modelo de producción y es quien responde a la competitividad. Con los recursos de afrontamiento, si la valoración cognitiva es positiva, generará bienestar y CVT; en caso contrario, en el sentido de la amenaza, se dará paso a la generación de los sintomas de estrés postulados por Lazarus y Folkman. ${ }^{7}$

En las sociedades que están en transformación permanente, lo normal es el desajuste y ajuste entre lo habitual y las nuevas condiciones de vida. En este contexto tienen mayor adaptación y bienestar aquellas sociedades que hayan desarrollado un sistema de predisposiciones, aptos y dispuestos para tomar decisiones en la incertidumbre, manteniendo la seguridad básica, aun cuando cambien drásticamente las circunstancias; lo primordial en esta reforma social es mantener una posición positiva, que busca el bienestar del individuo y por ende mejorar la calidad de vida. En este cambio de era las concepciones de los fenómenos sociales son holísticos, de integración sistémica; asimismo, se considera las decisiones democráticas, consensos y la negociación colectiva. ${ }^{13}$ El propósito del estudio fue analizar la relación entre la calidad de vida en el trabajo y los sintomas de estrés en el personal administrativo de una universidad pública del Ecuador. 


\section{METODOLOGÍA}

En el apartado de materiales y métodos se contempló el diseño metodológico, el mismo que se enmarcó en el paradigma cuantitativo, de corte transversal, porque estudió las variables simultáneamente en determinado momento haciendo un corte en el tiempo. Estudio descriptivo, observacional, analítico y relacional, porque buscó contestar por qué y cómo se relacionaron las variables de investigación, describiendo a los sujetos de estudio en su propia realidad y contexto, lo cual permitió generar conocimiento de la realidad objetiva de los trabajadores administrativos de la universidad pública del Ecuador. El estudio fue realizado con la anuencia de las autoridades de la institución, se incluyeron a sujetos que aceptaron participar, previa firma del Consentimiento Informado; además se amparó en el Código de Helsinki, Art. 13, 14 y 15, siendo el estudio de riesgo mínimo, donde prevaleció el criterio del respeto a su dignidad y protección de los derechos de los trabajadores.

Los criterios de inclusión de la población de estudio fueron trabajadores con nombramiento, con más de un año en el puesto de trabajo, de cualquier sexo. Se excluyeron a sujetos que no querían participar voluntariamente, que se encontraban con baja médica o con permiso. Se calculó la muestra aplicando la fórmula para poblaciones finitas. El muestreo fue bietápico: la etapa estratificada guardó proporcionalidad con la población de estudio y la aleatoria simple al interior de cada estrato, para que todos los sujetos tuvieran la misma probabilidad de formar parte del estudio. ${ }^{14}$

El procedimiento establecido para la ejecución de este trabajo investigativo, después de la anuencia de las autoridades de la universidad pública, fue aplicar una prueba piloto para calibrar los instrumentos, en una muestra de las mismas características de la población de estudio. Seguidamente se procedió a la realización del trabajo de campo, con el respectivo control y registro de las aplicaciones; a efectos de evaluar la variable independiente "calidad de vida en el trabajo" se aplicó el Cuestionario "CVT-GOHISALO" 3 , validado en población mexicana, que tiene una confiabilidad de 0,9527. Consta de 74 items, agrupados en siete dimensiones, a saber: Soporte Institucional (SIT), Seguridad en el Trabajo (ST), Integración al Puesto de Trabajo (IPT), Satisfacción por el Trabajo (ST), Bienestar Logrado a través del Trabajo (BLT), el Desarrollo Personal (DP) y la Administración del Tiempo Libre (ATL). El tipo de respuesta es en escala tipo Likert de 0 (peor calificación) a 4 (mejor calificación). Para la interpretación se consideró la escala de satisfacción baja, media y alta satisfacción en cada dimensión. Asimismo, para identificar la variable dependiente "presencia de síntomas de estrés", se aplicó el cuestionario de Evaluación del Estrés-Tercera Edición de Villalobos, ${ }^{14}$ validado en población colombiana, donde se calculó la correlación con las escalas de salud general y salud mental del cuestionario SF-36v2; ratificando la validez de constructo $(\mathrm{r}=87 ; \mathrm{p}=0,0001)$. Consta de 31 preguntas, que identifican cuatro categorías: Síntomas fisiológicos del estrés, sintomas de comportamiento social, sintomas intelectuales y laborales; y sintomas psicoemocionales. Los puntajes se interpretan a partir de la escala que ofrece las alternativas de muy bajo, bajo, medio, alto y muy alto. Se calificaron las pruebas, se estructuró la base de datos, vaciado y depuración de los mismos. Y, finamente, se realizó el análisis estadístico descriptivo e inferencial, porque predice, generaliza y aplica en el paquete estadístico SPSS 21. De la aplicación de los estadísticos de fiabilidad, se deduce el Alfa de Cron Bach de 0,830, considerado aceptable, así como la emisión y discusión de resultados, conclusiones, discusión y propuestas.

\section{RESULTADOS}

Del análisis descriptivo de la muestra de un total de 447 trabajadores administrativos de la universidad pública del Ecuador, se encontró que está representada por 182 hombres con un porcentaje de 40,7\% y 265 mujeres, correspondiente al 59,3\%. El promedio de edad es 46,62 años. El trabajador más joven tiene 26 años y el mayor 69 años de edad; estado civil casado (72,9\%), con dos hijos promedio; residen en vivienda propia el 71\% de ellos. La mitad de los trabajadores tienen un nivel de instrucción correspondiente a tercer nivel. El promedio de años en el cargo 18,63 años; perciben un sueldo entre 500 y 1.000 dólares. El Grupo Ocupacional es Servidor Público de Apoyo.

Para dimensionar cada uno de las dimensiones de la Calidad de Vida en el Trabajo (CVT), se determinaron las frecuencias y porcentajes, obtenidos en la población estudiada; de esta manera se desprenden los grados de satisfacción en loso niveles bajo, medio y alto, en cada dimensión de la CVT (Tabla 1).

En la dimensión Soporte Institucional en el Trabajo (SIT), se reflejó que el 43\% de los sujetos de estudio tiene un nivel bajo, lo cual implica que falta definir y evaluar los procesos de trabajo, así como una retroalimentación por parte del supervisor; al contrario de la tercera parte de esta población (30,6\%) que reflejó un nivel alto; y el 26,2\% que percibió un nivel medio de soporte institucional en el trabajo. Con respecto a la Seguridad en el Trabajo (ST), se apreció que solo una tercera parte de la población estudiada tiene una alta satisfacción en el lugar donde labora, es decir, percibe seguridad en el trabajo, tiene libertad de opinión, complacencia por la calidad y cantidad de los insumos y conformidad por el salario que percibe, de la misma manera, se encuentra en aquiescencia con los derechos contractuales. Las otras dos terceras partes de la población se encontraron en un nivel medio $(36,9 \%)$ y bajo de seguridad en el trabajo (24,6\%). En la dimensión Integración al Puesto de Trabajo (IPT), que engloba la pertinencia, la motivación y el ambiente de trabajo, más de la mitad de la población de estudio (64,2\%) tiene un nivel de satisfacción baja; y el 35,3\% repartido equitativamente en el nivel de satisfacción media y alta.

Tabla 1.

Frecuencias y Porcentajes de la Calidad de Vida en el Trabajo.

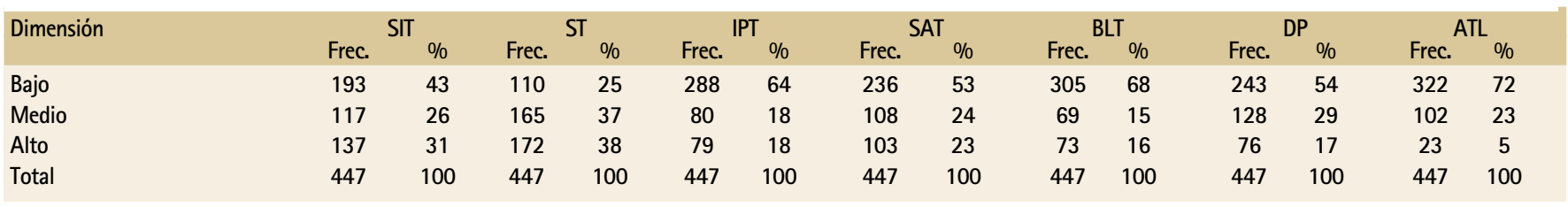


Tabla 2.

Frecuencia de las Dimensiones del Cuestionario de los Evaluación de Estrés.

\begin{tabular}{|c|c|c|c|c|c|c|c|c|}
\hline \multirow[t]{2}{*}{ Síntomas del estrés } & \multicolumn{2}{|c|}{ Sintomas Fisiológicos } & \multicolumn{2}{|c|}{$\begin{array}{l}\text { Síntomas de comportamiento social } \\
\text { (SCS) }\end{array}$} & \multicolumn{2}{|c|}{$\begin{array}{c}\text { Síntomas intelectuales y laborales } \\
\text { (SIL) }\end{array}$} & \multicolumn{2}{|c|}{ Síntomas Psicoemocionales } \\
\hline & Frecuencia & Porcentaje & Frecuencia & Porcentaje & Frecuencia & Porcentaje & Frecuencia & Porcentaje \\
\hline Muy bajo 1 & 53 & 11,9 & 285 & 63,8 & 149 & 33,3 & 397 & 88,8 \\
\hline Bajo 2 & 53 & 11,9 & 117 & 26,2 & 113 & 25,3 & 43 & 9,6 \\
\hline Medio 3 & 60 & 13,3 & 31 & 6,9 & 108 & 24,2 & 7 & 1,6 \\
\hline Alto 4 & 86 & 19,2 & 14 & 3,1 & 50 & 11,2 & 0 & 0 \\
\hline Muy alto 5 & 195 & 43,7 & 0 & 0 & 27 & 6 & 0 & 0 \\
\hline
\end{tabular}

En la Satisfacción por el Trabajo (SAT), 52,8\% tiene un nivel de satisfacción baja, lo cual manifiesta baja dedicación al trabajo, está ausente el sentimiento de orgullo por la institución, participación limitada en grupos, poca autonomía y percepción de que no hay reconocimiento y valoración por el trabajo realizado. El 24,2\% reflejó un nivel medio y un 23\% obtuvo un nivel de satisfacción alto en esta dimensión. En la dimensión Bienestar Logrado a través del Trabajo (BLT), más de la mitad de la población estudiada $(54,4 \%)$, manifestó un nivel bajo de disfrute de la actividad laboral que realiza. El 28,6\% de los trabajadores administrativos investigados reflejóuna media satisfacción es esta dimensión; y una minoría (17\%) mostró una alta satisfacción. En la dimensión Desarrollo Personal (DP), el 54,4\% reflejó un bajo nivel de satisfacción, es decir, que su percepción de los logros, expectativas de mejora y seguridad personal es mínima; el 28,6\% obtuvo un nivel medio; y el 17\% nivel alto de satisfacción en el desarrollo personal. En la Administración del Tiempo Libre (ATL), el 72\% tiene un nivel bajo de satisfacción, lo cual implica que no hay planificación, ni equilibrio entre trabajo y vida familiar; el 22,8\% obtuvo un nivel medio y, finalmente, el 5,1\% tiene un nivel alto de satisfacción en esta dimensión.

De la identificación de los síntomas de estrés, se desprendieron las siguientes frecuencias y porcentajes (Tabla 2).

El 76\% de los trabajadores administrativos advierte en los niveles muy altos y alto y medio la presencia de los sintomas fisiológicos de estrés, con prevalencia femenina en una relación de 2:1. En las demás dimensiones "Síntomas de Comportamiento Social (SCS), Síntomas Intelectuales y Laborales (SIL) y Síntomas Psicoemocionales (SP)», los niveles son bajos, lo que significa estabilidad en las relaciones familiares; cumplen con las normas de convivencia dentro de la sociedad, pueden iniciar sus actividades y permanecer en su puesto de trabajo, capacidad para concentrarse y ser creativos, no hay evidencia de signos o síntomas que refieran algún trastorno mental, emocional o consumo de sustancias y, además, perciben que en su puesto de trabajo no son propensos a sufrir accidentes laborales.

Para analizar la relación de las variables de investigación, se procedió a través de las tablas de contingencia a cruzar las frecuencias obser- vadas en las siete (7) dimensiones de la Calidad de Vida en el Trabajo (CVT), con las frecuencias observadas en las cuatro (4) dimensiones de los sintomas de estrés, obteniendo un total de veinte y ocho (28) tablas de contingencia. De las cuales se obtuvieron las frecuencias más sensibles, correspondientes a una baja calidad de vida y un alto o muy alto nivel de estrés (Tabla 3).

Del análisis de la relación de las variables de investigación, se apreció una prevalencia significativa en la contingencia de las dimensiones de CVT con la dimensión Síntomas Fisiológicos del Estrés (SF), lo que indica que la población estudiada tiene una baja calidad de vida y está relacionada con altos o muy altos manifestaciones de síntomas de estrés.

Para la prueba de hipótesis, el valor de $\mathrm{P}$ es menor a 0,05 , se rechaza la Ho, con el 95\% de confianza; y se prueba que existe evidencia de correlación significativa entre las variables; excepto en las correlaciones (SIT-SP), (ST-SP) y (ATL-SP).

Como el valor de $r$ está entre $-0,10$ y -0,25 la correlación entre las dimensiones de las variables es negativa débil, por lo que se aprueba la Hi, aceptando el vínculo entre las variables.

\section{DISCUSIÓN}

En este estudio se encontró que más de la mitad de los trabajadores administrativos de la universidad pública estudiada está en vulnerabilidad al riesgo del desequilibrio de la calidad de vida en el trabajo. Estos puntajes obtenidos tienen impacto en el ámbito laboral, personal y social. Se advierte la presencia de los síntomas fisiológicos de estrés, en un alto nivel, con prevalencia femenina en una relación de 2:1. Sin embargo, se descarta otro tipo de trastornos mentales o psicoemocionales.

La calidad de vida en el trabajo es catalogada como un tema coyuntural y trascendental para la salud ocupacional, por lo que hay que revalorizar el capital humano en respuesta a las exigencias de la globalización y la modernidad ${ }^{15}$, conceptos concordantes con Guerrero ${ }^{16}$ quien manifiesta que "los trabajadores están presentes como grandes articuladores del Modelo de Producción”

Tabla 3.

Relación de las dimensiones de las variables más sensibles de CVT y Síntomas de Estrés con frecuencias observadas.

$\begin{array}{lcccccc}\text { Dimensiones } & \begin{array}{c}\text { Soporte institucional Seguridad } \\ \text { para el Trabajo } \\ \text { (SIT) }\end{array} & \begin{array}{c}\text { en el trabajo } \\ \text { (ST) }\end{array} & \begin{array}{c}\text { Integración al } \\ \text { puesto de Trabajo } \\ \text { (IPT) }\end{array} & \begin{array}{c}\text { Satisfacción } \\ \text { por el Trabajo } \\ \text { (SAT) }\end{array} & \begin{array}{c}\text { Bienestar logrado } \\ \text { a través Trabajo } \\ \text { (BLT) }\end{array} & \begin{array}{c}\text { Desarrollo personal } \\ \text { Administración } \\ \text { del tiempo libre } \\ \text { (ATL) }\end{array} \\ \text { Sintomas fisiológicos (SF) } & 93 & 58 & 147 & 128 & 153 & 119 \\ \text { Sintomas de comportamiento social (SCS) } & 3 & 1 & 12 & 11 & 0 & 148 \\ \text { Sintomas intelectuales y laborales (SIL) } & 12 & 11 & 25 & 25 & 0 & 0 \\ \text { Sintomas Psicoemocionales (SP) } & 3 & 0 & 0 & 0 & 25 & 23 \\ \text { TOTAL } & 111 & 70 & 184 & 164 & 0 & 0 \\ \end{array}$


y añade que el desarrollo cultural de los trabajadores lleva a la humanización de los procesos de trabajo, así como a una nueva dimensión intelectual y de las relaciones sociales «en que el principal protagonista es el hombre, alrededor del cual deben crearse las mejores condiciones para el desempeño de su actividad laboral", es la manera de optimizar su calidad de vida en el trabajo. Chiavenato ${ }^{10}$ también se refiere al bienestar y la salud del trabajador como indispensables, para desempeñarse eficientemente en sus tareas. Sin embargo, estos argumentos se contraponen a los resultados de la investigación, donde más de la mitad de la población estudiada manifestó un bajo bienestar logrado a través del trabajo y no disfrutan de la actividad laboral.

Según el informe de evaluación del Sistema de Control Interno de la universidad pública (2011) los planes operativos que formulan las unidades académicas y administrativas no responden a un análisis detenido y suficiente que permita identificar las necesidades reales, para prever proyectos y satisfacer las demandas presentes y futuras; además, no se implementaron procedimientos de seguimiento y evaluación con indicadores de gestión, lo cual se evidenció en la dimensión de soporte institucional en el trabajo, lo que significa que los investigados perciben que falta definir y evaluar los procesos de trabajo. En este mismo sentido el Instituto Tavistock de Londres ha planteado un rediseño de puestos de trabajo, para que el empleado utilice sus destrezas y habilidades para el desarrollo personal. ${ }^{17}$

Argüelles ${ }^{18}$ concibe que el trabajador se integra al trabajo a través del empleo y, bajo su propia percepción «en su estudio aplicó el Cuestionario Calidad de Vida en el Trabajo CVT-GOHISALO», los resultados arrojados demostraron que más de la mitad de la población estudiada (Campeche, México) no se encuentra motivada, ni integrada al puesto de trabajo; se complementa con lo expuesto por Nadler y Lawler ${ }^{11}$, sobre la innovación del sistema de recompensas y mejorar las condiciones de trabajo y añaden que los trabajadores más comprometidos son los más motivados y satisfechos, postulados de los autores mencionados que no concuerdan con los resultados de esta investigación, en los que se revelan que más de la mitad de los sujetos estudiados no se identifican con su trabajo, ni están motivados, ni satisfechos. En el presente trabajo solo un 25\% de la población encuentra un nivel alto de satisfacción por el trabajo y refleja que faltan reconocimientos y valoración por el trabajo realizado. Constanza se refiere al sistema de recompensas para influir en el clima organizacional, la estabilidad en el empleo y el salario que reciben. ${ }^{19}$

León y Avargues ${ }^{20}$ identificaron la monotonía, falta de incentivos, promociones y poca adaptabilidad a la nueva tecnología en el personal administrativo en la Universidad de Sevilla, concluyendo que el acrecentamiento de la tensión generada por factores estresantes aumenta el nivel de estrés laboral. Datos semejantes a los encontrados en la presente investigación, donde la población estudiada tiene un promedio en 18 años en el cargo y poca adaptabilidad a los cambios tecnológicos, de la misma manera, el nivel de motivación es bajo.

En la dimensión administración del tiempo libre los resultados reflejaron que existe un nivel bajo de satisfacción, en la mayoría de la población estudiada. Es importante que exista un equilibrio entre trabajo y vida familiar; condiciones que no son nuevas; ya Aristóteles decía: "la cualidad de identificar al ocio con una condición o estado del ser, una condición del alma, que está divorciada del tiempo". Y desde otra visión objetiva, con una perspectiva cuantitativa, se conceptualiza como la porción del tiempo que sobra después de que el trabajo y los requerimientos de existencia han sido satisfechos. El trabajo es un medio que posibilita al ocio; el tiempo libre se asocia con el tiempo psicológico, es la calidad con que uno usa el tiempo. ${ }^{21} \mathrm{Al}$ respecto también Stebbins ${ }^{22}$ se pronuncia "de la combinación del ocio serio y casual, resulta un estilo de vida óptimo, de ocio cuyas actividades desarrollan el potencial humano y mejoran la calidad de vida".

Los resultados indicaron que pese a que la familia como el trabajo son fuentes de satisfacción no siempre convergen armónicamente y, por consiguiente, surge el conflicto trabajo-familia. Un estudio análogo sobre la interferencia del trabajo-familia realizado en la provincia de Santa Cruz de Tenerife reveló el conflicto percibido entre la vida laboral y familiar; pero le añade el componente "ocio", y comprobó que a mayor conflicto percibido en las tres dimensiones, las personas mostrarán un peor estado de salud general. ${ }^{23}$ Estos datos científicos difieren de los resultados obtenidos en la presente investigación, ya que demostraron que no hay dificultad en las relaciones familiares y trabajo, pese a que la distribución del tiempo libre no es equilibrada entre trabajo y familia.

La principal fortaleza del estudio es que hay una unificación de criterios, con respecto a la calidad de vida en el trabajo como un tema coyuntural y trascendente, por ende es ineludible que los trabajadores desarrollen sus capacidades individuales y de equipos, enfatizando en la concienciación para revalorizar el capital humano. Sin embargo, el estudio tiene varias limitaciones, ya que los trabajadores tienen recelo de participar o de identificarse, pensando en posibles represalias o por temor de ser removidos o perder el empleo y otros prefieren estar en la zona de confort.

El estudio aportado a la comunidad universitaria para concientizar sobre la necesidad de romper paradigmas hacia una nueva cultura organizacional, que se materialice más allá de las sensaciones y percepciones, que dignifique y desarrolle integralmente a los trabajadores, brindándoles oportunidades de promoción y progreso personal, asintiendo la multidimensionalidad de la calidad de vida en el trabajo. Los resultados del estudio, sin duda, permitieron plantear estrategias predictivas para mejorar la CVT en este contexto; y se alineó a la política de Estado que es promover el "buen vivir".

\section{CONCLUSIONES}

Las tres cuartas partes de la población estudiada advierten los sintomas fisiológicos del estrés en un alto nivel, lo cual es un indicativo de que su bienestar está en riesgo. Casi el cien por ciento de los trabajadores administrativos estudiados cumple con las normas de convivencia dentro de la sociedad y manifestó buenas relaciones interpersonales familiares. No evidenció ningún trastorno mental o psicoemocional.

Tres de cada diez empleados perciben el apoyo positivo de los superiores. La tercera parte de la población estudiada obtuvo una alta satisfacción en el trabajo. Más de la mitad de los trabajadores administrativos sino tienen pertinencia ni disposición para trabajar, $\mathrm{y}$ tampoco disfrutan de su trabajo, y la mayoría de los trabajadores no reflejó armonía y equilibrio entre el trabajo y la vida familiar.

Existe asociación positiva entre las variables: calidad de vida en el trabajo y síntomas de estrés en el personal administrativo de una universidad pública del Ecuador, considerando que el valor de $r$ está entre $-0,10$ y $-0,25$ la correlación entre las dimensiones de las varia- 
bles es negativa débil, por lo que se aprueba la $\mathrm{Hi}$, aceptando el vínculo entre las variables; se rechazó la Ho, con el 95\% de confianza; y se prueba que existe evidencia de correlación significativa entre las variables, excepto en las correlaciones: síntomas psicosomáticos tanto con soporte institucional y seguridad en el trabajo. El estudio es factible de replicarse en otras instituciones de educación superior similares con las adaptaciones pertinentes. Además, permitirá establecer programas de vida y planes de sucesión. Fomentando una red de apoyo social e induciendo a un cambio de paradigmas personales e institucionales acordes a la nueva era.

Partiendo del diagnóstico del recurso humano, instaurar programas de capacitación, promoción y un sistema de recompensas que mantengan motivados a los trabajadores y se mejore el ambiente laboral. Es decir, aplicar Talent Management. Promoviendo una cultura organizacional que se materialice más allá de las sensaciones y percepciones, dignificando al trabajador, asintiendo la multidimensionalidad de la CVT.
Sin duda, la investigación permitió plantear estrategias predictivas para mejorar en el contexto universitario la CV; y a su vez, se alineó a la política de Estado que es promover el "buen vivir".

\section{Agradecimientos}

Se deja constancia de agradecimiento a la Universidad de Guadalajara, al Centro Universitario de Ciencias de la Salud y al Departamento de Salud Pública. Así como al Cuerpo Tutorial y grupo de Sinodales, que aportaron con su contingencia para la realización de esta investigación.

El tema fue presentado en una ponencia en el 1er Congreso Internacional de Gestión y Desarrollo de Organizaciones Saludables y Sustentables, en los Valles, Guadalajara, el 17 y 18 de Octubre de 2013.

\section{REFERENCIAS}

1. Warr P. The measurement of well-being and other aspects of mental health. J Occup Psychol. 1990; 63(3):193-210

2. Lawton M. The dimensions of morale. In: Kent DP, Kastenbaum R, Sherwood $S$, editors. Research, planning and action for the elderly. New York: Behavioral Publications, Inc.; 1972. p. 144-165

3. González R, Hidalgo G, Salazar J, Preciado M. Instrumento para medir la calidad de vida en el trabajo CVT-GOHISALO. Guadalajara: Instituto de Salud Ocupacional; 2009

4. Seligman, M. Aprenda optimismo. Haga de la vida una experiencia maravillosa. Barcelona: Grijalbo.; 1998.

5. Walton RE. Conciliación de conflictos interpersonales. México: Fondo Educativo Interamericano; 1973.

6. Sosa E. Qué es el estrés ocupacional, enfermedades derivadas y reconocidas por la legislación Colombiana. Med segur trab. [en línea] 2011 [citado feb 2013].Disponible en: file:///C:/Users/Mercy/Downloads/Dialnet-QueEsEIEstresO cupacionalEnfermedadesDerivadasYReco-3677388\%20(1).pdf.

7. Lazarus R, Folkman S. Estrés y procesos cognitivos. Barcelona: Ramírez Roca; 1984.

8. Matteson MT, Ivancevich J. Controlling Word Stress. London: Jossey-Bass; 1987.

9. Cohen S, Kessler RC. Measuring stress: A guide for health and social scientists. New York: Oxford University Press; 1977.

10. Chiavenato I. Higiene, seguridad y calidad de vida. En: Chiavenato I. Gestión del talento humano. Bogotá: McGraw Hill; 2004.p. 36.

11. Nadler DA, Lawler EE. Factors influencing the success of labor management quality of work life projects. J Occup Behav. 1983; 1(1): 53-67.

12. Leka $S$, Griffiths $A, C o x T$. La organización del trabajo y el estrés. Ginebra: OMS; 2004. (Protección de la salud de los trabajadores; 3 ).

13. Kerr H, Schumann M. Limits of the Division of Labour: New Production and Employment Concepts in West Germany Industry. Econ Ind Democracy. 1987;8:251-170

14. Villalobos M. Estudio de una Bateria de Instrumentos para la evaluación de factores psicosociales. Bogotá: Pontificia Universidad Javeriana; 2010.
15. Granados I. Calidad de vida laboral: historia, dimensiones y beneficios. IIPSI [en línea] 2011; 14(2): 271-276 [citado 2 may 2013]. Disponible en: http:/l sisbib.unmsm.edu.pe/bvrevistas/investigacion_psicologia/v14_n2/pdf/ a14v14n2.pdf.

16. Guerrero J, Cañedo $R$, Rubio $S$, Cutiño $M$, Fernández $D$. Calidad de vida y trabajo: algunas consideraciones en el ambiente laboral de la oficina. ACIMED [en línea]; 2006;14(4):0-0[cited 14 jun 2013]. Available from: http://www.studymode.com/ essays/Richard-Lazarus-And-Susan-Folkman-s-And-960622.html.

17. Navarrete F. Calidad de vida laboral. Revista de la Universidad del Valle de Atemajac. 2005;19 (51):18.

18. Arguelles L. Propuesta de modelo predictivo de la Calidad de Vida Laboral en el sector Turistico Campechano. Global Conference on Business and Finance Proceedings. San José, Costa Rica, 28-31 Mayo 2013. IBFR. 2013;8(3).

19. Constanza, B. \& Peralta, A. Liderazgo, Clima y Satisfacción Laboral en las Organizaciones. Universum. 2007; 22 (2):40-56

20. León JM, Avargues ML. Evaluación del estrés laboral en personal universitario. Mapfre Medicina. 2007;18(4):323-332.

21. Hernández A, Morales V. Una revisión teórica: ocio, tiempo libre y animación sociocultural.EFdeportes.com [en línea] 2008;13(127):00 [citado 23 feb 2013]. Disponible en: http://www.efdeportes.com/efd127/una-revision-teorica-ociotiempo-libre-y-animacion-sociocultural.htm

22. Stebbins E. Amateurs, Professionals, and Serious Leisure. Montreal: Mcgill Queens University Press; 1992.

23. Pérez F. Familia, Estrés Laboral y Calidad de Vida: Resultados Preliminares de un Estudio Piloto en la Provincia de Santa Cruz de Tenerife.Poster [en línea] [citado dic 2013]. Disponible en: http://www.cop.es/colegiados/T-00921/ poste-familia.pdf

\section{Referencia complementaria:}

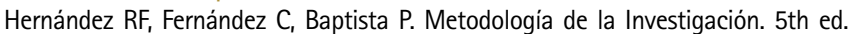
México: McGraw-Hill; 2010. 